\title{
Labyrinthe
}

$5 \mid 2000$

Numéro 5

\section{Administrer l'identité}

Le premier âge des papiers d'identité en France (XVIII ${ }^{\mathrm{e}}$-milieu XIX siècle)

\section{Vincent Denis}

\section{(2) OpenEdition}

\section{Journals}

Édition électronique

URL : http://journals.openedition.org/labyrinthe/258

DOI : $10.4000 /$ labyrinthe. 258

ISSN : 1950-6031

Éditeur

Hermann

Édition imprimée

Date de publication : 15 janvier 2000

Pagination : 25-42

Référence électronique

Vincent Denis, "Administrer l'identité », Labyrinthe [En ligne], 5 | 2000, mis en ligne le 16 février 2005, consulté le 19 avril 2019. URL : http://journals.openedition.org/labyrinthe/258 ; DOI : 10.4000/

labyrinthe.258

Ce document a été généré automatiquement le 19 avril 2019.

Propriété intellectuelle 


\title{
Administrer l'identité
}

\author{
Le premier âge des papiers d'identité en France (XVIII ${ }^{\mathrm{e}}$-milieu XIX ${ }^{\mathrm{e}}$ \\ siècle)
}

\section{Vincent Denis}

1 L'utilisation de documents écrits, produits par l'administration, pour prouver notre identité, nous semble aujourd'hui aller de soi. Dans l'histoire de la société française, cet usage courant est cependant une invention tardive, et s'est lentement répandu, par des seuils successifs qu'il n'est pas aisé de repérer, depuis seulement deux ou trois cents ans. Le siècle qui court de la fin du règne de Louis XIV au Premier Empire a été ainsi le théâtre de transformations essentielles dans le régime de l'identification des individus. Se pencher sur cette période c'est mettre en lumière les origines intellectuelles et administratives de pratiques de classement et d'identification traditionnellement associées à des époques plus tardives. En effet, on assiste alors à l'élaboration de plusieurs techniques d'identification, en l'espace de quelques décennies, qu'il s'agisse du billet de congé ou du passeport imprimé, des registres de signalement normalisés ou encore du livret ouvrier. C'est là aussi en l'espace de quelques décennies, que l'État civil est placé sous le strict contrôle de la justice royale, dès 1736 . Tandis que l'usage des documents d'identité se développe et se codifie et que leurs formes s'institutionnalisent, on assiste à une montée continue d'exigences nouvelles en matière d'identification, plus précises, reposant sur l'écrit et de nouvelles catégories descriptives. Qu'est-ce qui a rendu possible l'émergence de ces nouvelles formes d'identification "par le papier » au dix-huitième siècle ? Par quels cheminements se diffusent-elles dans le tissu social ? On tentera ici de répondre à ces questions, tout en dégageant les seuils successifs du développement des « identités de papier $»^{*_{1}}$.

$2 \mathrm{Au}$ début du siècle, l'usage des papiers pour prouver son identité reste relativement limité. Certes on connait alors certaines institutions d'identification, mais leur utilisation n'a rien d'une règle générale. Assurément il existe des "passeports ", et depuis plusieurs siècles. Le passeport est alors défini comme une permission et une recommandation donnée à un individu ou à un groupe pour se déplacer. Ils sont employés par quelques voyageurs, et surtout en temps de guerre, notamment pour voyager en sécurité dans les 
régions frontalières. En dehors de ces cas, on s'en munit assez rarement. Du côté de l'État civil, toute une législation royale, et particulier l'ordonnance criminelle de 1667, prescrit la tenue des registres de baptême, mariages et sépultures, dans chaque paroisse, afin de produire des preuves de « l'état » d'un individu devant la justice, en cas de besoin. Mais ces modes restent encore peu utilisés, et l'ordonnance de 1667 permet encore l'utilisation de la preuve par témoins si on ne peut prouver son identité par des documents écrits. Par ailleurs, ce que l'on sait de l'état des registres et en particulier de l'obligation du dépôt d'un double exemplaire au greffe du siège de la justice royale dans chaque bailliage laisse penser que le recours est peu fréquent, voire impraticable dans de très nombreux cas, étant donné la mauvaise tenue des registres, ou leur mauvais état de conservation.

3 Cet état de fait se transforme progressivement à partir des décennies 1720-1730, où se produit une inflexion, introduisant de la part des autorités royales et policières un souci plus grand d'identifier par le papier.

4 De ce point de vue, l'administration militaire semble avoir joué un rôle pionnier, en développant de manière systématique l'utilisation de techniques d'identification perfectionnées, dans les premières décennies du dix-huitième siècle. L'ordonnance royale du 2 juillet 1716 créé d'une part les contrôles des troupes de l'armée française. Ces registres sont établis " pour faciliter à l'avenir la recherche des déserteurs et avoir une connaissance plus particulière des cavaliers, dragons et soldats dont les compagnies seront composées ", et chacun contiendra "dans les colonnes marquées sur ledit registre, les noms propres, de famille et de guerre des sergens, caporaux, anspessades et soldats desdites compagnies, le lieu de leur naissance, l'élection, bailliage, seneschaussée ou chatellenie, dans le ressort desquels ledit lieu sera situé, leur âge, leur taille, les marques qui peuvent servir à les faire reconnaître et les dates de leur enrollement " (art. XXII). D'autre part elle institue l'utilisation de petits formulaires imprimés à remplir, délivrés par les officiers à chaque soldat libéré, attestant qu'il n'est pas un déserteur. Les officiers doivent « spécifier dans le corps desdits congés, le païs, l'âge, la taille, la couleur des cheveux ou de la perruque, et les autres signes qui pourront faire reconnoitre les soldats pour lesquels ils seront expédiés, de manière qu'ils ne puissent servir pour d'autres que pour eux ", ce qui fait de ces congés (ou cartouches, comme on dit à l'époque) de véritables petits documents d'identité individuels. C'est en fait dans ces deux registres - constitution d'un corpus fiable servant de référence à l'identité des individus, et élaboration de titres d'identité individuels - que les progrès sont considérables et accélérés à partir des années suivantes.

On constate en effet un recours plus impérieux aux papiers à partir des années 1720-1730, en particulier dans un certain nombre de grandes villes du royaume. La lutte contre l'errance s'articule ici à un souci nouveau d'identification et de transparence de la part de certaines élites urbaines. L'identification devient synonyme de repérage et de classification des individus. Un témoin de cette inflexion brutale semble être la nouvelle politique de lutte contre la mendicité inaugurée par la déclaration royale de 1724. Elle lance de nouvelles opérations d'" enfermement " contre les mendiants, errants et vagabonds qui infestent le royaume, sous l'égide de l'autorité royale. Ce type de campagne n'est pas nouveau, surtout depuis la création de l'Hôpital Général en 1656. Elle fait suite cependant aux épisodes répressifs de la Régence, entre 1719 et 1722, pendant lesquels on avait confié à une compagnie privée chargée de la mise en valeur de la Louisiane, la Compagnie du Mississippi, le soin d'arrêter mendiants et vagabonds pour les " transporter " vers les colonies françaises ${ }^{2}$. Les hommes de main de la Compagnie avaient multiplié les arrestations brutalement et sans distinction, les opérations avaient 
dégénéré en émeutes à Paris en 1720 , et le gouvernement royal avait mis fin à l'expérience. La nouvelle politique inaugurée à partir de 1724 se caractérise par un souci nouveau d'identification précise des cibles de la répression, et de la définition de leur traitement, variable selon leur statut, la gravité des actes, leur sexe, leur condition physique et leur âge. La déclaration du 18 juillet 1724 montre ainsi l'élaboration de procédures d'identification et de classement pour identifier et arrêter les mendiants, reposant sur les papiers, les signalements et les marques. Outre une classification des cibles de la répression, elle institue une codification des procédures de contrôle des papiers. C'est la possession d'un titre valide - passeport non expiré et dont l'itinéraire est bien respecté, et par lequel l'autorité qui l'a délivré répond du porteur et garantit son honorabilité, ou congé prouvant qu'on est bien un ancien soldat - qui va permettre au suspect d'échapper à l'arrestation par la maréchaussée. Même si cette politique n'est appliquée qu'entre 1724 et 1733, elle marque l'irruption d'un esprit nouveau et la diffusion de techniques nouvelles d'identification. Elle montre aussi que les appareils de contrôle et de répression contribuent fortement au développement des papiers d'identité. C'est par touches successives que l'on peut voir se diffuser leur usage et la montée des exigences de leur utilisation. Celles-ci sont beaucoup plus manifestes dans la deuxième moitié du dix-huitième siècle.

La lutte contre l'errance, reprise avec une vigueur nouvelle à partir de 1768 avec la création des dépôts de mendicité, joue le rôle d'un accélérateur. La maréchaussée quadrille les campagnes, multiplie les descentes dans les lieux de rassemblement, foires, marchés ou sites de pèlerinages, pour attraper à coup sûr mauvais sujets et vagabonds. À chaque réaffirmation de la volonté royale d'éradiquer la mendicité et le vagabondage, elle redouble de zèle. Pour la maréchaussée, c'est un faisceau d'indices qui permet de tracer la ligne de séparation entre le pauvre et le vagabond dangereux, dans lesquels la possession de papiers prend une importance croissante, comme le montrent les arrestations effectuées sur un lieu de pèlerinage important en Bourgogne ${ }^{3}$. Claudien Cambillier, vendeuse de livres de dévotion et d'images pieuses à deux sous, est libérée grâce aux factures en sa possession, qui prouvent qu'elle est une marchande de profession, et aux certificats de son curé. Il est encore moins difficile de se procurer un passeport, même sans disposer de recommandations. La plupart des grandes villes abritent un cabaretier douteux prêt à témoigner pour quelqu'un, attestant le connaître, ainsi que ses affaires dans le lieu, et pourquoi il a besoin d'emprunter la route qu'il désire prendre. Doublet, traiteur à Chartres, a agi ainsi pour tous les membres de la bande d'Orgères, un groupe de brigands célèbre des dernières années du dix-huitième siècle. Plus facile à falsifier ou à obtenir, le passeport ne tend pas moins à voir son usage s'accroître. L'aveu reste un moyen privilégié, mais surtout pour se justifier en cas d'arrestation ou de contestation sérieuse par les autorités. Le passeport est le document essentiel des contrôles et des déclarations de routine, qui ont la faveur des autorités policières et urbaines, et dont le développement contribue également à " fabriquer de l'identité de papier " par touches successives.

7 Dans les organismes urbains importants, le « contrôle des étrangers » se développe. Chaque ville a son histoire, mais la tendance commune est à la rationalisation et à la systématisation de dispositifs plus anciens. La surveillance des " étrangers » en ville, c'est-à-dire des individus qui n'y sont pas domiciliés, repose sur les déclarations des logeurs, aubergistes ou des habitants qui les hébergent : à Toulouse ou à Lille les formulaires doivent être déposés tous les soirs au siège de la policé ${ }^{4}$. À Paris des 
inspecteurs contrôlent régulièrement les registres des établissements, tandis que dans les années 1760 est inventé le système de la " fiche d'hôtel », destinée à la police ${ }^{5}$. Chacun est sommé de décliner son identité, mais surtout son lieu de résidence en ville. L'efficacité des dispositifs va croissant, comme leur recours au papier : à Lille, la consommation de ces papiers imprimés pour toutes les portes de la ville est de 6 mains en 1693 : en 1786 elle est passée à 12 mains rien que pour les deux portes les plus importantes.

C'est dans un contexte plus général de volonté de mise en ordre des populations et de l'espace urbain que s'affirme l'usage des papiers. Cette tendance est particulièrement visible dans les dernières décennies de l'Ancien Régime, à partir de la fin des années 1760 . La « mise en carte », jusque-là réservée aux militaires, considérés comme des déserteurs potentiels, s'étend à d'autres pans de la population, par étapes successives.

Les ouvriers en sont la principale cible. À Paris, les compagnons, les patrons et les communautés de métier sont en lutte permanente pour le contrôle du marché du travail au dix-huitième siècle 6 . Il s'agit pour les communautés de mettre fin aux prétentions des compagnons à diriger le placement des ouvriers, mais aussi de lutter contre les " travailleurs en chambre ", employés clandestinement par des patrons qui n'appartiennent pas aux communautés de métier, notamment dans le faubourg SaintAntoine. Émerge aussi une suspicion croissante contre les " mauvais ouvriers ", remuants, indisciplinés, et les associations compagnonniques promptes à boycotter les ateliers qui ne paient pas assez. Elle s'accompagne d'un désir de fixer la main d'œuvre. Le soupçon contre l'errance, le vagabondage et l'indiscipline des ouvriers, cher à la police, rejoint ici les considérations économiques et les intérêts stratégiques des communautés, dans un projet de contrôle de la main d'œuvre qui s'appuie sur un nouveau type de carte. Le précédent pilier du système fut longtemps le congé écrit, certificat délivré par le maître à son compagnon et qu'il doit présenter pour obtenir un nouvel emploi. La formalité, ancienne, est réaffirmée avec une vigueur nouvelle en 1749 , puis maintes fois répétée ensuite, dans un contexte d'agitation croissante. Au lendemain de la suppression puis du rétablissement des communautés en 1776, une nouvelle technique et un nouveau document voient le jour, synthétisant le modèle militaire que l'on a déjà vu repris pour les mendiants : le livret ouvrier. Il s'agit, comme le prescrivent les statuts de la communauté des couvreurs de Paris, pour tous les compagnons « d'aller se faire inscrire au Bureau de la Communauté [...] d'y déclarer leurs noms, surnoms, âge, le lieu de leur naissance et celui de leur domicile actuel, comme aussi le nom du Maître chez lequel ils travailleront alors; et pour ceux qui seront sans Attelier ou Boutique, ou qui arriveront à Paris, le nom du dernier Maitre chez lequel ils ont travaillé, soit à Paris, soit en Province, enfin le genre de travail qu'ils ont adopté ; laquelle déclaration sera inscrite par le Commis qui sera par Nous préposé, ainsi que le signalement du Compagnon, sur un Registre tenu à cet effet audit Bureau ». Le commis délivrera alors « un Livret ou petit Registre 7 ». La mesure, adoptée à Paris dès 1778-1779, est étendue à tout le royaume en 1781 , sans que le signalement soit généralisé cependant. À la fois document d'identité et certificat de bonne conduite, le livret garantit en théorie l'insertion de l'ouvrier dans le circuit normal du travail, tout en permettant son contrôle et son identification.

On voit ainsi se multiplier les formes d'identification et les papiers d'identité, délivrés par une multitude d'institutions. De ce point de vue, la diversité des pouvoirs sous l'Ancien Régime, le souci d'ordre qui en anime certains, et le recours croissant aux papiers contribuent ensemble à la floraison des documents divers attestant l'identité de leur 
porteur, du simple certificat signé par le curé aux livrets ouvriers les plus sophistiqués, en passant par les différents types de passeports et de congés.

11 Cette profusion des formes tient d'abord à la diversité de leurs statuts. Les passeports sont des actes qui ont valeur de recommandation, et par lesquels l'autorité qui les délivre demande à ce que leur porteur ne soit pas inquiété sur sa route. De fait, toute " autorité ", qu'il s'agisse du curé, d'un officier municipal ou de police, ou d'un ministre du roi, est habilitée à en expédier. Qu'il s'agisse de franchir les frontières ou d'effectuer un voyage dans le royaume, le voyageur prudent s'en munit, d'autant plus que les contrôles se multiplient, au moins pour les catégories les plus humbles de la population. Un individu sans papiers, incapable de se faire " avouer ", c'est-à-dire qu'une institution ou une personne, à travers le certificat, atteste de sa respectabilité (et de son identité), s'expose à ne plus pouvoir donner de gages objectifs ou acceptables de son honorabilité, et donc à être sérieusement inquiété. Quant aux congés, certificats et livrets, mêmes délivrés à des individus dans des situations spécifiques, ils restent recevables comme les autres documents d'identité en l'absence d'une norme bien établie.

Mais cette profusion n'empêche pas au cours de la deuxième moitié du dix-huitième siècle certains modèles, certains codes de circuler et de s'imposer peu à peu. Le modèle élaboré pour les militaires par l'ordonnance du 2 juillet 1716, comprenant notamment le signalement du porteur, et l'adoption pour les registres d'un formulaire standardisé, identifiant avec les mêmes catégories tous les individus, est imité pour l'identification des mendiants et des vagabonds dans l'ordonnance de 1724, puis encore lors de la mise en place du livret ouvrier au tournant des années 1770. La façon dont les signalements sont rédigés évolue également dans le sens d'une standardisation, comme on peut le voir dans les contrôles de troupes de l'armée tout au long du dix-huitième siècle ${ }^{8}$ : l'usage de formulaires imprimés, généralisé dans les années 1760, entraîne l'élimination des caractéristiques les plus singulières des signalements de chaque soldat : des expressions comme " menton à la Henry quattre » ou "bègue » disparaissent, au profit de termes neutres, impersonnels et interchangeables. La codification des descriptions physiques, selon des catégories canoniques (visage, front, yeux, nez, bouche, menton) et des qualificatifs standards (rond, allongé, ou simplement « moyen » pour la forme du visage), permet alors le classement des individus ainsi enregistrés - c'est ce que l'on réalise dès 1724 avec les signalements des mendiants arrêtés ${ }^{9}$ - au prix de l'effacement des caractères idiosyncrasiques de chacun. Seuls certains éléments particulièrement saillants sont encore notés dans les "signes particuliers ", notamment les cicatrices visibles. Une nouvelle définition de l'identité émerge, les hommes n'étant plus caractérisés par l'existence de traits physiques irréductiblement individuels, mais par une certaine combinaison de traits puisés dans un répertoire de possibles. Une telle conception a pour avantage de permettre la classification, mais elle s'éloigne de la pratique réelle de la reconnaissance des individus, fondée sur les traits particuliers, et dans laquelle excellent les bons « physionomistes » comme les sergents recruteurs. Surtout, elle se condamne à aller droit dans une impasse théorique et pratique, privilégiant ce qui rapproche les hommes, en rendant finalement tous les caractères physiques « moyens » et les individus à peu près semblables physiquement. Il faudra attendre les découvertes anthropométriques de Bertillon et les travaux de Galton sur les empreintes digitales dans le dernier tiers $d u x^{e}{ }^{e}$ siècle, mettant au jour l'existence de traits physiques irréductiblement individuels et non modifiables, pour sortir de cette définition. 
13 Si les codes de l'individualité s'élaborent peu à peu, de nombreux obstacles s'opposent à une standardisation des documents d'identification. À la prolifération des institutions qui les délivrent, en l'absence de norme centralisée (à quelques exceptions près, comme les congés militaires ou, dans une certaine mesure, les livrets ouvriers), s'ajoutent les pesanteurs d'une société hiérarchisée, où la diversité et l'inégalité des conditions des hommes interdisent par exemple qu'on puisse aligner sur la même page d'un registre et de la même façon un jeune cadet de la noblesse et un domestique. Les considérations de statut, contribuent ainsi à soustraire certains groupes aux formes nouvelles d'identification. Vers la fin du dix-huitième siècle, dans l'Almanach Royal, sorte de bottin administratif avant la lettre, les nouvelles formes de présentation des corps parisiens et de leurs membres, en particulier le classement alphabétique, sont perçues comme des atteintes aux hiérarchies traditionnelles. D’une manière analogue, le numérotage des maisons à Paris, à l'initiative de l'éditeur d'un guide de Paris, se heurte à pendant quelques années à l'opposition farouche du Procureur général du Parlement de Paris, Joly de Fleury ${ }^{10}$. À la méfiance des privilégiés pour ce qui peut donner " un air d'égalité » s'ajoute une répugnance parfois manifeste des hommes du dixhuitième siècle pour certaines formes d'identification comme le signalement physique. Cette technique, utilisée au départ pour rechercher les déserteurs et les criminels, est longtemps jugée infamante. Sous l'Ancien Régime, il n'est pas concevable d'y soumettre des " gens de qualité ». Dans les contrôles des troupes, les signalements disparaissent à mesure que l'on s'élève dans la hiérarchie, et disparaissent pour les officiers. Un corps d'élite comme les Suisses en sera toujours exempté11. Dans les passeports, forme la plus ancienne qui semble rester imperméable aux influences du modèle militaire jusqu'à la Révolution, l'identification des personnes varie selon leur statut, et se fait selon des catégories encore longtemps peu homogènes ${ }^{12}$. Le moyen de préciser l'identité était d'abord d'indiquer un nom et un prénom, éventuellement le titre de noblesse. Le lieu de naissance ou le lieu de résidence sont parfois indiqués. Le statut social figure fréquemment : il peut s'agir d'une qualité (" gentilhomme »), d'une profession (marchand, artisan...), d'un office ou d'une charge. La nationalité est confondue dans cette catégorie : on parle d'un « gentilhomme saxon » ou d'un " confiturier allemand ». La mention « dame » ou " damoiselle » signale l'appartenance au sexe féminin. En revanche, le nom reste obscur pour les hommes de Dieu, qui gardent leur nom de religion. Les passeports d'Ancien Régime étaient souvent délivrés, dans le cas d'un déplacement collectif, au nom du ou des voyageurs les plus importants. Les accompagnants pouvaient se dispenser de passeports, et on les retrouve mentionnés d'une façon plus ou moins vague sur le même passeport. Sur celui-ci, le porteur signale les « hardes » qu'il emporte, mais aussi ses valets - qui ne sont jamais nommés ou décrits, sauf exception comme un « valet nègre » -, sa famille - femme ou enfant, avec parfois l'identité d'un parent -, le personnel dont s'entourent certaines professions - " garçons ", notamment pour les marchands de chevaux, " commis » ou " hommes d'affaires » pour les négociants - ou encore les escortes, armées ou non, pour les princes et les dignitaires, enfin les gouverneurs - parfois nommés - pour les jeunes gens venant faire leurs " exercices ». La tendance est de ne pas nommer le personnel domestique : d'après Lucien Bély, la meilleure façon de voyager incognito était de se faire passer pour un domestique.

14 Si les nouvelles formes d'identification par le papier tendent à connaître un usage plus répandu, elles ne sont qu'un élément d'un système qui repose encore largement sur le recours aux témoins, et sur des notions comme la « réputation » et la « moralité », où ce 
que l'on voit et ce que l'on entend importe plus que ce que l'on lit, quand on aborde un inconnu. Nous avons affaire à des sociétés où l'interaction entre les individus est surtout fondée sur l'interconnaissance, sans la médiation du document écrit. Le voyageur, hors des structures d'accueil comme la famille ou le métier, est bien vite un inconnu, et dans certaines circonstances, il peut devenir un suspect. Les autorités des corps de ville, la police comme la maréchaussée, dans leur soupçon contre l'errance, peuvent alors le rejeter dans la catégorie des " gens sans aveu », c'est-à-dire de ceux qui sont sans attaches et sans état attestés. Pour déchirer le voile d'étrangeté qui l'entoure, l'inconnu doit pouvoir se faire "avouer ». Les passeports, billets de congé et autres certificats n'ont pas le caractère impersonnel de nos documents d'identité contemporains. Ces documents sont moins des preuves d'identité que des certificats de respectabilité. Par leur biais, l'autorité qui les délivre atteste à distance de l'honorabilité et de l'identité de leur porteur. L'aveu direct semble d'ailleurs parfois préférable aux documents écrits, comme le montre l'examen des individus arrêtés par la maréchaussée sur le pèlerinage de Reineen-Auxois dans les années $1760^{13}$. Un couple venu d'Italie, formé d'un compagnon chapelier sans travail et d'une autre marchande de littérature de dévotion, parvient à se faire élargir en combinant la présentation de factures, du congé militaire de l'homme (qui a servi jusqu'à l'année précédente dans un régiment) et la possibilité de se faire « avouer » : c'est ainsi que la mère du compagnon se manifeste pour le faire libérer. Inversement, on rencontre le cas de vendeurs aux ressources plus limitées qui ne pourront convaincre la maréchaussée qu'ils ne sont pas des mendiants : malgré un certificat de son curé, un infirme venu acheter quelques objets de dévotion pour les revendre est ainsi envoyé au dépôt de force d'Avallon. On voit donc que les papiers ne constituent encore ni la seule preuve d'identité ni la plus importante. L'aveu reste préféré au passeport en dernier ressort, car il est moins ouvert aux fraudes ${ }^{14}$. En être dépourvu est assurément une preuve de vagabondage. L'aveu est cependant une porte ouverte aux abus : les curés auvergnats soutenaient ainsi leurs ouailles dans leurs odyssées, et accordaient des certificats de bonnes mœurs sans discrimination. Le curé d'ollières était réputé pour cela dans toute la province. Le gouverneur du dépôt de mendicité de Riom, Henrion de Bussy, se plaignait régulièrement de lui. Olwen Hufton cite un exemple typique : « rien de plus inconsidéré et de plus léger que les curés. Celui des Ollières, qui vraisemblablement a fait ses études aux Jésuites, ose signer et certifier à Monsieur l'intendant que Borie s'est bien comporté dans sa paroisse, qu'il en est absent depuis quelque temps. Quelque temps, c'est depuis 5 ans et le jeune homme n'en a que vingt. Ce jeune homme courre et libertine fortement depuis cinq ans dans tout le Languedoc et à grand frais. Il nous vient de Montpelier ; et ce bon curé avait oublié non seulement de faire faire la première communion à son paroissien mais même de lui apprendre, à la lettre, le signe de la croix».

15 En définitive, se conjuguent pour faire obstacle à l'émergence d'une forme d'identification rationnelle l'absence d'uniformité législative et administrative, l'inégalité des conditions, la répugnance face des formes d'identification jugées infamantes ou indiscrètes, l'attachement à une logique du face-à-face et de l'apparence. S'il ne faut pas dissimuler les avancées de la période révolutionnaire et surtout de la période napoléonienne, ces obstacles semblent reculer parfois difficilement, ou perdurent. La continuité entre les tendances évoquées plus haut et les pratiques d'identification des premières décennies du dix-neuvième siècle est frappante.

Utilisant ou synthétisant des techniques d'identification élaborées dans les dernières décennies de l'Ancien Régime, la Révolution ne peut être considérée comme une véritable 
rupture. Certes, elle permet de faire sauter quelques-uns des obstacles à l'avènement de formes uniques. L'unification juridique et administrative de la nation, l'égalité devant la loi, l'abolition des titres, enfin le brouillage conséquent des codes sociaux et hiérarchiques qui régissaient la société d'Ancien Régime, sont autant de facteurs favorables à l'émergence d'une forme unique d'identification. Les autorités révolutionnaires puis le Directoire accordent une attention renouvelée à la surveillance et au repérage des individus. Les circonstances troublées, l'agitation politique, la peur des complots, la guerre enfin attirent l'attention des gouvernants sur la nécessité de maintenir le système des passeports, rétablis par le décret du 28 mars 1792 après une éphémère abolition. Le rêve de transparence de l'utopie révolutionnaire rejoint ici les considérations sécuritaires pour vaincre les résistances de ceux qui voient dans les passeports un vestige de la tyrannie. Ce souci de transparence devient dès le Directoire un souci de surveillance, mais il n'en accélère pas moins le développement des papiers d'identité. Cette tendance n'aboutit pas cependant à la standardisation des formes d'identification. D'un côté la croissance d'une administration longtemps décentralisée dans le choix de ses moyens entraîne l'apparition de nouveaux documents hétéroclites comme les cartes de sûreté municipales - réalisés selon des modèles assez divers. Si la loi sur les passeports du 28 mars 1792 réserve aux mairies et préfectures le droit de délivrer des passeports, et définit un formulaire au niveau national, chaque administration départementale est libre de la fabrication de ses feuilles de passeports, du support aux caractères, jusqu'à l'Empire. Si certaines catégories de titres disparaissent - comme les livrets ouvriers, avant d'être rétablis par loi du 22 germinal an XI (12 avril 1803), ou les nombreux certificats délivrés par les curés par exemple - on est loin d'une véritable rationalisation. La multiplicité des modèles de documents, de cachet, d'encres, de papier et de caractères employés facilite la tâche aux fraudeurs de toute sorte - émigrés ou opposants politiques, bandits, déserteurs, réfractaires.

L'idée d'uniformité elle-même fait difficilement son chemin. Jusqu'au Directoire, les autorités sont dépassées par la fraude, et ne se détachent pas de l'idée qu'un formulaire unique soit une garantie de contrôle suffisant. Le rattrapage technique par rapport aux fraudeurs n'a lieu que pendant la décennie suivante, sous le Consulat et l'Empire. Il faut plusieurs années pour tirer les leçons de la production massive par l'État de titres et actes imprimés (pensons aux assignats) et de leur falsification, qui explosent l'une et l'autre pendant la décennie révolutionnaire. Le poids des événements - il a souvent fallu improviser, avec des expédients - a probablement bloqué les administrateurs dans cette tâche. Une réflexion technique s'engage sur la fraude et l'identification des individus dans les dernières années du Directoire. De cet effort on conserve quelques produits : des mémoires adressés aux gouvernements. Celui d'un certain Ducrest est tout entier travaillé par un idéal de transparence, à la fois du territoire et du corps social ${ }^{15}$. Il prône l'établissement d'un système de passeports individuels, délivrés sur la base d'un recensement annuel de tous les habitants de l'ensemble des bâtiments du pays, par un corps spécial de " dénombrateurs ». Il suggère une refonte des limites administratives, sur une base mathématique, et surtout la numérotation des départements, des districts, des communes, et des maisons. Chaque habitant serait ainsi identifié et localisable par une suite de numéros, reportée sur son passeport, avec son signalement et un cachet spécial du département pour faire bonne mesure. Jamais réalisé, ce projet, typique d'une statistique démographique qui est encore asservie aux finances et à la police, n'en révèle pas moins un dessein de surveillance absolue et rationnelle, où les administrateurs combinent sans états d'âme contrôle policier et recensement. Plus concrètement, une 
commission au Conseil des Cinq-Cents, l'une des deux chambres, travaille à une réforme du système des passeports vers 1798-1799. Ses travaux n'aboutissent pas avant la chute du régime, mais elle a permis l'examen de projets dont les éléments pourront être repris plus tard. C'est le cas notamment du Mémoire théorique et pratique sur les moyens d'assurer la police des passeports dans toute la République d'un certain Bonet de Treyches, que Fouché, ministre de la Police général de Napoléon Bonaparte, ressort des cartons de ses archives vers $1804^{16}$. Ce mémoire a pour originalité de prétendre assurer la sécurité du système des passeports et de garantir l'identification des individus en jouant seulement sur le support et la forme des passeports, à l'inverse des lois et des textes réglementaires qui ne s'y intéressaient guère. Le mémoire, qui peut se lire aussi comme un véritable bréviaire contre la contrefaçon, discute du choix du papier, des caractères d'imprimerie, des encres, de l'emploi des couleurs. Il présente les avantages de l'adoption d'un papier à filigrane dit "sympathique ", dont toute altération sera visible. L'auteur du mémoire prône aussi l'emploi d'un procédé d'impression d'un genre nouveau, et l'utilisation de techniques sophistiquées comme le "stéréotypage ", soit la fusion en un seul bloc de caractères mobiles, permettant la reconnaissance d'un passeport par sa simple superposition avec un autre. Bonet propose finalement des applications illimitées à son procédé coûteux mais efficace : l'impression des cartes de sûreté, certificats de vie et de résidence, cartouches, congés et brevets militaires, lettres de marque, billets de loterie... Il suggère de rationaliser ainsi l'ensemble de la production imprimée de titres et attestations délivrés par l'État. Le mémoire de Bonet préfigure ainsi le rattrapage technique de l'État napoléonien dans sa lutte avec les faussaires et contrefacteurs.

Dans l'immédiat, c'est l'idée de réaliser un modèle de passeport, pour voyager sur le territoire français ou à l'étranger, infalsifiable et surtout uniforme pour l'ensemble du territoire national qui intéresse Fouché. La réforme engagée débouche alors sur la mise en place d'un tel système, pour la première fois en France, à partir de 1807. Pour la police, l'ensemble des documents d'identité forme un tout cohérent, et Fouché puis ses successeurs s'efforcent de faire respecter l'obligation de s'en munir, pour rendre chaque individu identifiable. Cette idée est résumée par la déclaration de Réal, un des adjoints de Fouché : comme l'entrepreneur doit se munir d'une patente, l'ouvrier d'un livret, le soldat d'une feuille de route, chaque voyageur doit avoir un passeport. L'ensemble de la population est ainsi assujetti selon son statut à un type de document. Le livret ouvrier a été rétabli dès 1803 , nanti d'un signalement obligatoire du porteur, sans pour autant dispenser les ouvriers d'un passeport. La période napoléonienne coïncide donc avec une rationalisation croissante des formes d'identification : celles-ci sont limitées à quelques grands types, au sein desquels les passeports occupent une place privilégiée. La logique du face-à-face perdure cependant : la présentation de témoins pour obtenir un passeport reste obligatoire, même si la présentation d'autres preuves d'identité - en particulier écrites - progresse, sans toutefois toujours s'y substituer. L'action de la police pour faire respecter le port obligatoire des passeports, notamment pour les passeports intérieurs (pour tout individu voyageant hors de son canton, depuis la loi sur la police des communes de vendémiaire an IV) n'a pas été non plus sans mal. On peut cependant penser qu'elle a accoutumé les populations à l'usage des « identités de papier ». L'administration napoléonienne bâtit en quelques années un système qui lui survit pendant plusieurs décennies. Il n'est abandonné qu'à la fin du Second Empire et au début de la Troisième République. Entre-temps, il est vrai, la France est entrée dans l'âge industriel, et les techniques dont les bases intellectuelles ont été élaborées entre les dernières décennies 
de l'Ancien régime et le Premier Empire ne sont plus adaptées à l'ère du chemin de fer, du télégraphe et de la photographie.

Si certains obstacles à une identification rationnelle subsistent, la période étudiée n'en est pas moins traversée par quelques évolutions essentielles. La rationalisation progressive des techniques d'identification, accélérée pendant la Révolution et le Consulat en est une : les passeports napoléoniens sont bien loin des certificats rédigés à la main par les curés de campagne. Ce mouvement s'appuie aussi sur les progrès enregistrés dans la tenue de registres d'identification, comme l'État civil et les contrôles des troupes. Le dix-huitième siècle voit le contrôle et le soin dont ils font l'objet, s'accroître, tandis que se produit leur mise sous la tutelle exclusive de l'État, dès la Déclaration royale du 17 juillet 1736, avant même que la Révolution ne les confie aux maires. Enfin s'impose le recours croissant à ces documents, à des couches toujours plus larges de la population, non pas seulement pour contrôler et surveiller, mais dans les relations avec les administrations, ne serait-ce que pour accéder à un emploi ou bénéficier d'un droit. En définitive c'est une période cruciale pour la mise en place des techniques d'identification, comme pour l'apprentissage des gestes et des pratiques liées aux papiers. Il est aussi probable que les populations soumises à ces nouveaux usages modifient leur définition même des caractères constitutifs de l'individualité. C'est à travers ces cheminements que s'acclimate la notion d'identité individuelle, mentionnée pour la première fois dans le Code civil.

\section{BIBLIOGRAPHIE}

CORVISIERAndré, Les Contrôles de troupes de l'Ancien Régime : Guide des recherches, Vincennes, Service Historique de l'Armée de Terre, 1968.

GUTTON Jean-Pierre, L'État et la mendicité dans la première moitié du XVIII siècle : Auvergne, Beaujolais, Forez, Lyonnais, Lyon, Institut d'études foréziennes, 1973.

HUFTON Olwen, « Begging, vagrancy, vagabondage and the law : an aspect of the problem of poverty in eighteenth-century France », European Studies Review, 2, 1972, p. 97-123

KAPLAN Steven Lawrence, «La lutte pour le contrôle du marché du travail à Paris au XVIII ${ }^{\mathrm{e}}$ siècle », Revue d'histoire moderne et contemporaine, 1989, p. 361-412.

NOIRIEL Gérard, « Surveiller les déplacements ou identifier les personnes ? Contribution à l'histoire du passeport en France de la I ${ }^{\mathrm{re}}$ à la III ${ }^{\mathrm{e}}$ République ", Genèses, n 30, mars 1998, p. 77-100. NoRDMAN Daniel, « Sauf-conduits et passeports », dans BÉLY Lucien (s.d.), Dictionnaire de l'Ancien Régime, Paris, Presses Universitaires de France, 1996.

SCHWARTZ Robert M., Policing the Poor in Eighteenth-Century France, Chapel Hill ; London, University of North Carolina Press, 1988.

VIDALENC Jean, « Une source d'histoire économique et sociale : les passeports. Problèmes d'utilisation, limites et lacunes ", C.T.H.S., Bulletin de la Section d'Histoire moderne et contemporaine, 8,1971, p. $187-202$. 


\section{NOTES}

1.* Cet article s'inscrit dans le cadre de la préparation d'une thèse de doctorat en histoire, consacréeà l'identification des individus en France de 1715 à 1815, sous la direction d'Alain Cabantous à l'Université de Paris I.

2.Cf. R.M. Schwartz, Policing the Poor in Eighteenth-Century France, Chapel Hill ; London, University of North Carolina Press, 1988.

3.Cf. Dominique Julia, «Les pèlerins de Sainte Reine au XVIII ${ }^{\mathrm{e}}$ siècle », dans Ph. Boutry et D. Julia (dir.) Reine au Mont Auxois : le culte et le pèlerinage de Saine Reine des origines à nos jours, Cerf ; Ville de Dijon, 1997, p. 269-271.

4.Cf. J.-L. Laffont, Policer la ville: Toulouse capitale provinciale au siècle des Lumières, Thèse de doctorat, Université de Toulouse-Le Mirail, 1997, et C. Clémens-Denys, La Sécurité urbaine dans les villes de la frontière du Nord au XVIII e siècle, Thèse de doctorat, Université d'Artois, 1997. 5.Cf. J.-C. Dubost, «Une mobilité spécifique : les étrangers à Paris au siècle des Lumières ", dans Étrangers et provinciaux à Paris, XVII $e_{-X V I I I}^{e}$ siècles, sous la dir. de Daniel Roche, à paraître aux Éditions Rue d'Ulm.

6.Sous l'Ancien Régime, la communauté de métier est un corps, avec sa réglementation et ses juges, qui regroupe tous ceux qui exercent un « métier », une activité artisanale déterminée dans une ville. Sur les conflits parisiens, voir S. L. Kaplan, « La lutte pour le contrôle du marché du travail à Paris au XVIII siècle », Revue d'Histoire moderne et contemporaine, 1989, p. 361-412.

7.Archives Nationales, AD XI 16, ordonnance de police du 19 juin 1779.

8.Cf. A. Corvisier, Les Contrôles de troupes de l'Ancien Régime: Guide des recherches, Vincennes, Service Historique de l'Armée de Terre, 1968, en particulier le tome premier, Une source d'histoire sociale.

9.Cf. J.-P. Gutton, L'État et la mendicité dans la première moitié du XVIII siècle : Auvergne, Beaujolais, Forez, Lyonnais, Institut d'études foréziennes, Lyon, 1973.

10.Cf. J. Pronteau, Les Numérotages des maisons de Paris du XVe siècle à nos jours, Ville de Paris, 1966.

11.Cf. A. Corvisier, op. cit.

12.Voir l'étude de 2000 passeports délivrés par le Ministère des Affaires Étrangères en 1712, dans L. Bély, Espions et ambassadeurs au temps de Louis XIV, Paris, Fayard, 1990, IV ${ }^{e}$ partie, chap. 4.

13.Cf. D. Julia,art. cit.

14.Cf. Olwen Hufton, « Begging, vagrancy, vagabondage and the law : an aspect of the problem of poverty in eighteenth-century France », European Studies Review, 2, 1972, p. 97-123.

15.Archives Nationales, F7 4283.

16.Archives Nationales, F7 4279. 


\section{RÉSUMÉS}

Le XVIII ${ }^{\mathrm{e}}$ siècle voit se développer des techniques d'identification fondées sur le papier et l'écrit. Malgré la multiplicité des titres et des supports utilisés, des codes descriptifs de l'individualité s'élaborent progressivement ; cependant les obstacles à une identification rationnelle des individus restent nombreux : la hiérarchie et l'inégalité des conditions, la répugnance qu'inspirent des pratiques jugées infamantes ou indiscrètes, ou encore le poids de la logique du face-à-face, de l'attestation par témoins, au détriment des formes impersonnelles d'identification écrite. La Révolution et la période napoléonienne précipitent la centralisation et l'uniformisation du système. Mais la continuité l'emporte, l'identification se faisant jusqu'au dernier tiers du XIX ${ }^{\mathrm{e}}$ siècle sur des bases intellectuelles élaborées au XVIII ${ }^{\mathrm{e}}$ siècle, et selon des techniques mises en place entre le Directoire et le Premier Empire. Ces systèmes, rudimentaires comparés à ceux mis en place à partir de la Troisième République, n'en ont pas moins diffusé au sein des populations de nouveaux gestes, de nouveaux codes de l'identité, désormais fondée sur le papier.

Ruling identity. The origins of identification papers in France from the eghteenth century up to the middle of the nneteenth century.- the Eighteenth Century, some techniques of identification, based on paper and writing, are developed. Despite the multiplicity of titles and forms used, descriptive codes of individuality are progressively drawn up. However, the impediments to a rational identification remain numerous : the hierarchy and the inequality of conditions, the repugnance inspired by practices considered as defamatory or indiscreet, the weight of the logics of face to face, of affidavits, to the detriment of impersonal forms of written identification. The Revolution and the Napoleonic period accelerate the concentration and the unification of the system. Still, continuity prevails : indeed, up until the last third of the century, identification is based on the intellectual concepts formed in the eghteenth Century and on certain techniques developed between the Directoire and the First Empire.

\section{AUTEUR}

\section{VINCENT DENIS}

Vincent DENIS, né en 1972, ancien élève de l'École normale supérieure, agrégé d'histoire, enseigne, en tant qu'AMN, l'histoire moderne à l'Université de Paris I (PanthéonSorbonne). Il prépare une thèse de doctorat consacrée à l'identification des individus en France, de 1715 à 1815. 